\title{
Bibliography
}

G. K. Ackermann and J. Eichler, Holography: A Practical Approach, Wiley-VCH, Berlin (2008).

S. A. Benton and V. M. Bove, Jr., Holographic Imaging, Wiley Interscience, Berlin (2008).

H. J. Caulfield, The Art and Science of Holography: A Tribute to Emmett Leith and Yuri Denisyuk, SPIE Press, Bellingham, WA (2004).

H. J. Caulfield, Handbook of Optical Holography, Academic Press, New York (1979).

J. Goodman, Introduction to Fourier Optics, 3rd ed., Roberts and Company Publishers, (2004).

R. K. Kostuk, Holography: Principles and Applications, CRC Press, Boca Raton, FL (2012).

Y. G. Soskind. Field Guide to Diffractive Optics, SPIE Press, Bellingham, WA (2011) [doi: 10.1117/ 10.1117/3.895041]. 
Index

aberration, 35

aberrations in holograms

(paraxial

approximation), 76

accommodation, 58

acousto-optic modulator

(AOM), 70

Airy disk, 29

amplitude gratings, 21

amplitude/phase, 16

angular dispersion, 13, 73

angular multiplexing, 44,

\section{1, 53}

atmospheric scattering, 58

binary/digital/analog, 16

blaze curve, 23

bragg's law, 14, 51, 73

charge-coupled device

(CCD), 56

coherence length, 73

coherent, 5-6

coherent waves, 5-6

complementary metal-

oxide semiconductor

(CMOS), 56

complex notation, 3

computer-generated

hologram, 10, 37, 76

conjugated term, 33

coupled-wave analysis, 14, 21

dichromated gelatin

(DCG), 64

difference in writing and

reading wavelengths,

35

diffracted beams, 34

diffraction, 7

diffraction by a slit, 29 diffraction by apertures, 75

diffraction efficiency of thick volume gratings, 74

diffraction efficiency of

thin gratings, 74

diffraction grating, 9-10

diffraction integrals, 75

diffraction pattern, 8

diffraction terms from a

hologram, 76

digital holography, 56

digital micromirror device

(DMD), 71

edge-lit hologram, 18, 48

efficiency of a digitized-

phase hologram, 76

efficiency of multiplexed

holograms, 76

electric field, 2

embossed hologram, 66

energy distribution, 14

Fourier holograms, 37

Fourier/Fresnel, 16

Fraunhofer diffraction

integral, 28

Fresnel diffraction

integral, 27

Fresnel holograms, 38

Fresnel transformation,

56

Fresnel zone plate, 10, 30

Gabor zone plate, 30

gelatin, 64

generalization in two

dimensions, 29

Gerchberg-Saxton

algorithm, 39 


\section{Index}

grating equation, 12-13, $31,35,73$

grating vector, 15

Helmholtz equation, 2, 73

hologram, 8

holographic data storage, 49

holographic interferometry, 54

holographic optical elements (HOEs), 10

holographic printing, 49 holographic stereograms, 49

holographic television, 57 image sensor, 56 inline point sources, 32 inline reflection hologram, 43

inline transmission

holograms, 42 inorganic crystals, 69 interference, 4 interference visibility, 73 interferogram, 4 intermodulation, 33 intermodulation noise, 34 $\mathrm{K}$-vector closure condition, 15

Kirchhoff diffraction integral, 26

LCoS, 71

linear perspective, 58

Lippmann photography, 52

liquid crystal on silicon (LCoS), 71

lookup tables, 38 magnetic field, 2 master hologram, 45

media thickness variation, 36

micro-opto-electromechanical systems (MOEMS), 71 movement parallax, 58 multicolor images, 50 multiple slits, 30 multiplexing, 53 object beam, 34 occlusion, 58 off-axis transmission hologram, 44 on-axis/off-axis, 16 optical field, 2 optical time reversal, 55 optical/computational, 16 orders, 9 organic compounds, 69 orthoscopic image, 45 perceptual factors, 58 phase conjugate mirror, 55 phase conjugation, 55 phase gratings, 20 phase or transmittance discarded, 39 phase shifting, 56 phase stabilization

system, 60 photochromic materials,

65

photopolymer, 63 photorefractive materials, 68-69 photoresists, 66 physiological factors, 58 piston-mirror MOEMS, 72 plane wave, 2,73 


\section{Index}

plane waves incident at different angles, 31 polarization holograms, 67 position vector, 2 pseudoscopic, 45 pulsed laser, 61 quantization, 40 rainbow holograms, 47, 51 reading beam, 34 real pseudoscopic image, 34

reference beam, 34 reflection geometry, 17 reflection hologram, 52 reflection/transmission, 16 remarkable-thin-grating, 30

resolution, 58 rigorous coupled-wave analysis (RCWA), 21-22

sampling, 40 sawtooth-function phase, 25

shading, 58 side-by-side point sources, 32

silver halide, 52, 62 sinusoidal phase, 24 sinusoidal transmittance, 24

slant angle, 14 source spatial extent, 36 space-bandwidth product (SBP), 41, 57, 76 spatial light modulator (SLM), 71 spherical wave, 3,73 square-function phase, 25 square-wave

transmittance, 24 stereoscopic parallax, 58 super-blaze, 23 surface/volume, 16 transfer hologram, 45-46 thick grating condition,

$$
\text { 20-21 }
$$

thick gratings, 19 thick-volume gratings, 23 thin gratings, 19, 24 thin/thick, 16, 73 transfer hologram (H2), 46

transmission geometry, 18

transmission holograms, 45 transmission stereograms, 51

two-beam interference patterns, 75

two-plane-wave interference, 73

vergence, 58 virtual orthoscopic image, 34 visibility, 4 wave vector, 2 wavefront, 8 wavelength multiplexing, 53 


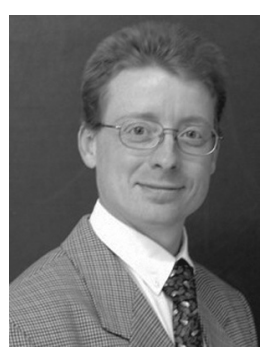

Pierre-Alexandre Blanche is an Assistant Research Professor at the College of Optical Sciences, University of Arizona. He received his Ph.D. in 1999 from the University of Liège (Belgium), after which he held a postdoctoral position at the University of Arizona in the field of nonlinear optics. Back in Belgium, he developed a hologram production facility for diverse scientific applications using dichromated gelatin and photopolymers. In 2006, he joined the College of Optical Sciences to work on photorefractive materials, holographic $3 \mathrm{D}$ displays, and various diffractive optical systems. 